A. Pramesh Rao, G. Swarup and Gopal-Krishna, eds.

\title{
High dynamic Range, Interferences Tolerant, Digital Receivers for Radioastronomy: Results and Projects at Paris and Nanay Observatory
}

\author{
Carlo Rosolen, Alain Lecacheux, Eric Gerard, Vincent Clerc \\ Observatoire de Paris-Meudon, CNRS 5 Place Jules Janssen. 92195 \\ Meudon cedex., France
}

Laurent Denis

Station de Radioastronomie de Nancay, 18330 Nancay, France

\begin{abstract}
Radio astronomy in the decameter to centimeter wavelength range is facing new challenges because of man made interferences due to increasing needs in telecommunications. At the Radioastronomy department of Paris Meudon Observatory, we have been working since four years on high dynamic range digital receivers based on Digital Signal Processors (DSP). The first achievement is a digital spectro- polarimeter devoted to spectroscopy of astrophysical radiation in decameter range, now in operation at the Nancay Decameter array. The block diagram of the receiver includes a high dynamic range analogue section followed by a 12 bits analogue to digital converter. The digital part makes use of high power, programmable digital circuits for signal processing, arranged in a dedicated parallel architecture, able to compute in real time the power spectrum and the correlation of the input signals. This receiver was also used, as spectrometer backend, at Nancay decimetric radiotelescope and has performed very well in the presence of very strong interferences. We are presently working on a new digital receiver with broader bandwidth. The objective is $2 \times 25 \mathrm{MHz}$ band with at least $60 \mathrm{~dB}$ dynamic range. This new receiver will use additional computation power in order to recognise and avoid man made interferences which corrupt the radio astronomical signal. At the Nancay Radioastronomy Observatory, we have started to develop a new digital configurable receiver with 8 times $25 \mathrm{MHz}$ band and ten thousand channels. For low frequency radioastronomy, direct spectrum computation technique is really powerful and offers new capabilities for real time interferences excision. Fig. 1 shows pulsar observations in the presence of interference made with the DSP receiver on the UTR2 radiotelescope. Fig. 2 shows the effect of satellite interfernce on $\mathrm{OH}$ observations made with the Nancay telescope. Fig. 3 shows the block diagram of the DSP system and demonstrates how offline excision of interference in the frequency time-domain enables recovery of the signal. The final spectrum had 960 minutes integration on and off source and took 8045 minutes of procession on a $450 \mathrm{MHz}$ Pentium II.
\end{abstract}




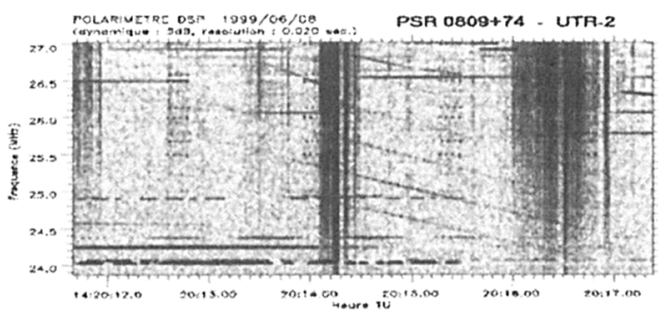

Figure 1. Real time detection of dispersed pulses of PSR $0809+74$ in presence of strong RFI with DSP receiver at UTR-2 decameter radiotelescope. (Lecacheux, Konovalenko et al., in prep.)
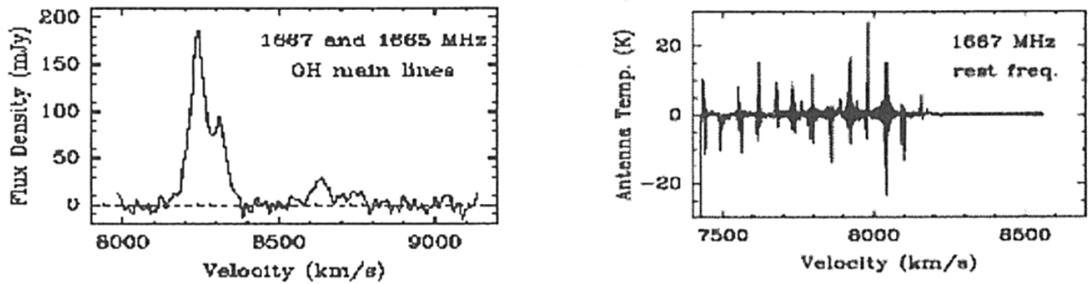

Figure 2. III ZW 35 IRAS seen with NRT before and after Iridium
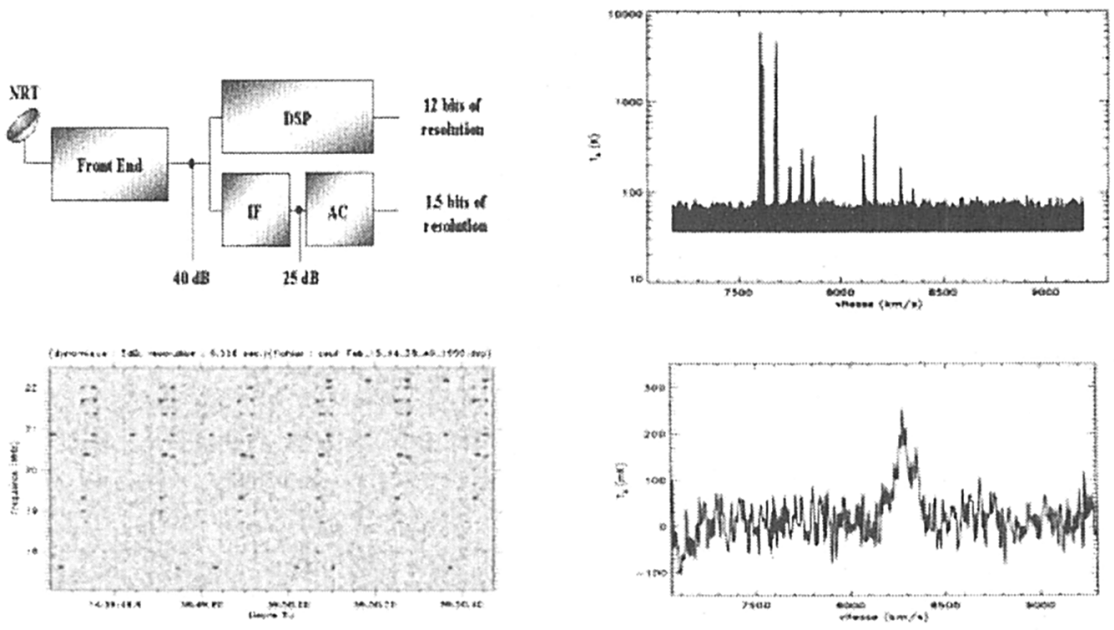

Figure 3. (a) Description of measurements with DSP receiver. (b) Raw spectra, after correction of the receiver spectral response at $12 \mathrm{~ms}$ resolution shows that Iridium is 10000 stronger than the sky noise level. (c) frequency-time diagram. (d) final spectrum after interference excision 